\title{
Southampirstrof
Son
}

Optoelectronics

Research Centre

\section{Record High Capacity (6.8 Tbit/s) WDM Coherent Transmission in Hollow-Core Antiresonant Fiber}

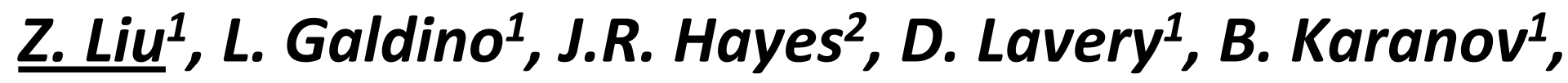
D.J. Elson ${ }^{1}$, K. Shi ${ }^{1}$, B.C. Thomsen ${ }^{1}$, M.N. Petrovich ${ }^{2}$, D.J. Richardson' ${ }^{2}$, F. Poletti ${ }^{2}$, R. Slavík ${ }^{2}$, and P. Bayvel ${ }^{1}$

1. Optical Networks Group, Dept. of Electronic \& Electrical Engineering, UCL(University College London), UK

2. Optoelectronics Research Centre (ORC), University of Southampton, UK

Photonic Hyperhighway 


\section{Hollow-Core Fiber}

\section{Guide light in air rather than solid material}

- Ultimately low latency (50\% faster than silica fiber)

- Ultra-low nonlinearity (1000 times smaller than SMF-28)

- High damage threshold (handle up to Giga Watt)

- Low temperature sensitivity (>100 times lower than SMF-28)

Hollow-core Photonic Bandgap Fiber Hollow-core Antiresonant Fiber

\section{(HC-PBGF) (HC-ARF)}

\section{Highest data rate:}

73.7 ala

\section{[V.A.J.M.}

32(4), 201

Longest

74.

NOT About

HC-PBGF

[M. Kuscherov, et al., ECOC, 2015]

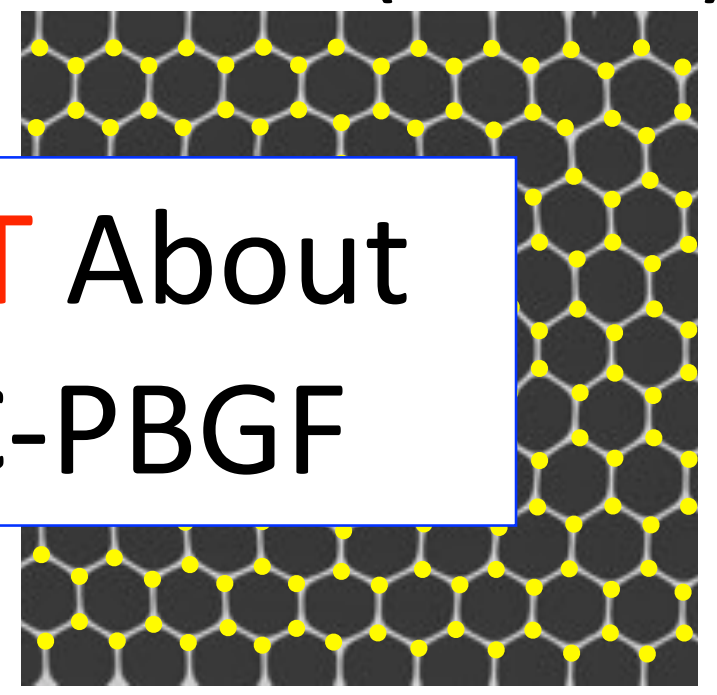

Lattice Pitch

decides performance

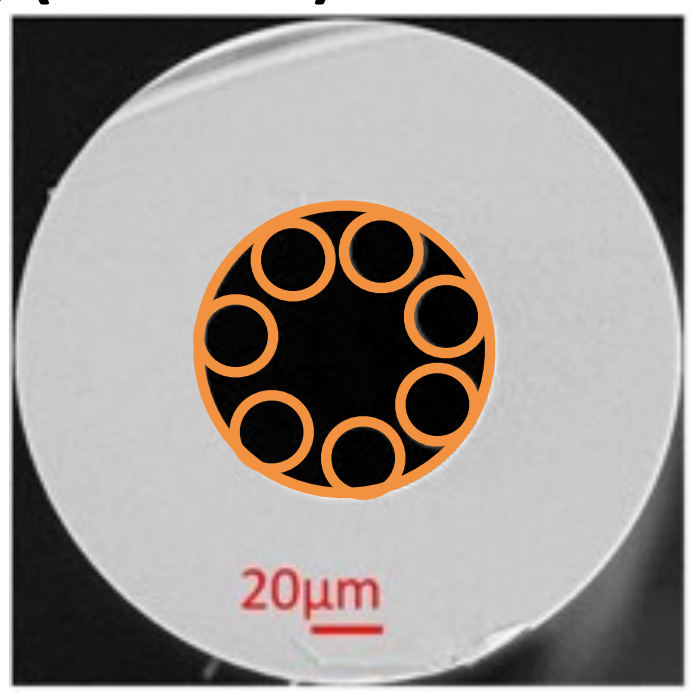

Highest data rate: $10 \mathrm{~Gb} / \mathrm{s}$

Longest Distance: 100 meters

[J. Hayes, et al., JLT, 35, 2017]
Thickness of the thin wall decides performance 
Fiber Structure

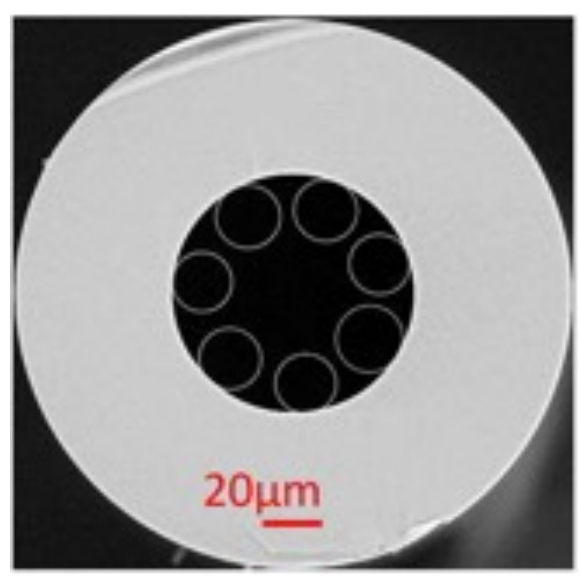

Mode field image (high mode purity)

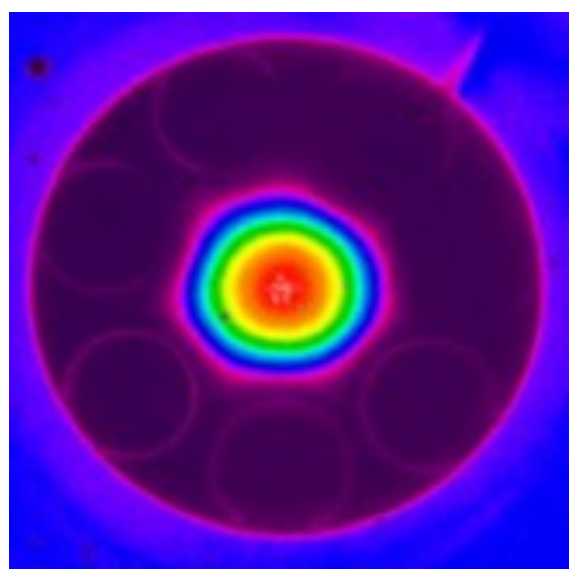

Special features can only get from HC-ARF

- High mode purity with large core

- Broadband guidance (over $1100 \mathrm{~nm}$ )

- Low dispersion ( \pm 2 ps across entire band)

- Simple structure (easy fabrication)

- Potential low loss $(0.1 \mathrm{~dB} / \mathrm{km})$ with nested structure

F. Poletti, Opt Express 22, 23807 (2014) 
Length:

Data transmission:

Loss:
100 meters

[J.R. Hayes et al., JLT, 35, 2017.]

$10 \mathrm{~Gb} / \mathrm{s}$ OOK

[J.R. Hayes et al., JLT, 35, 2017.]

$7.7 \mathrm{~dB} / \mathrm{km}$ (at $750 \mathrm{~nm}$ )

[B. Debord, et al., Optica, 4, 2017.]

\section{We show:}

- Dual-polarization high-order formats transmission

- WDM transmission

- Comparison with SMF-28 in nonlinearity penalty 
Fiber Used in This Experiment

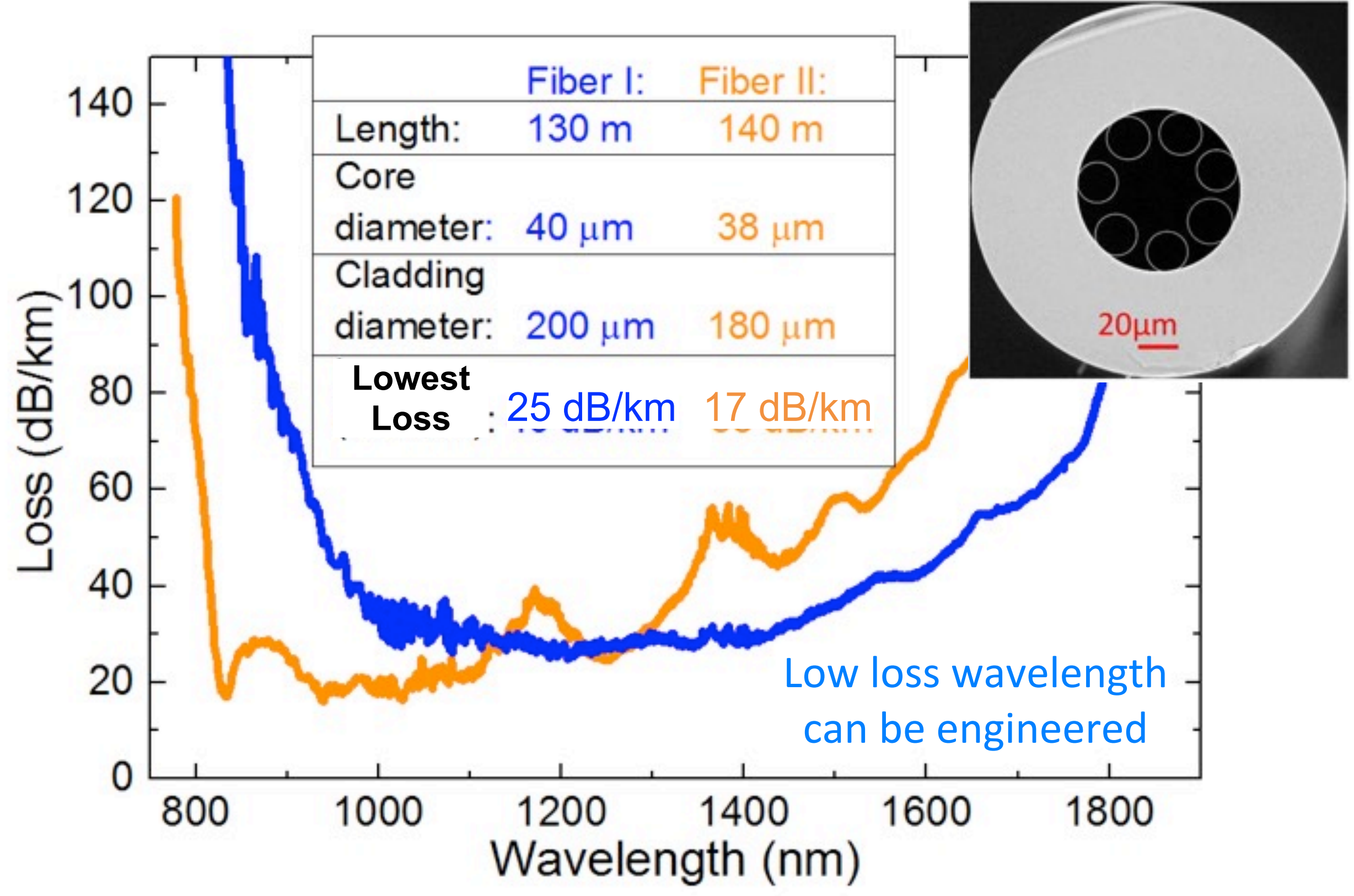


Splice Image: SMF28 - ARF Splice Image: ARF - ARF

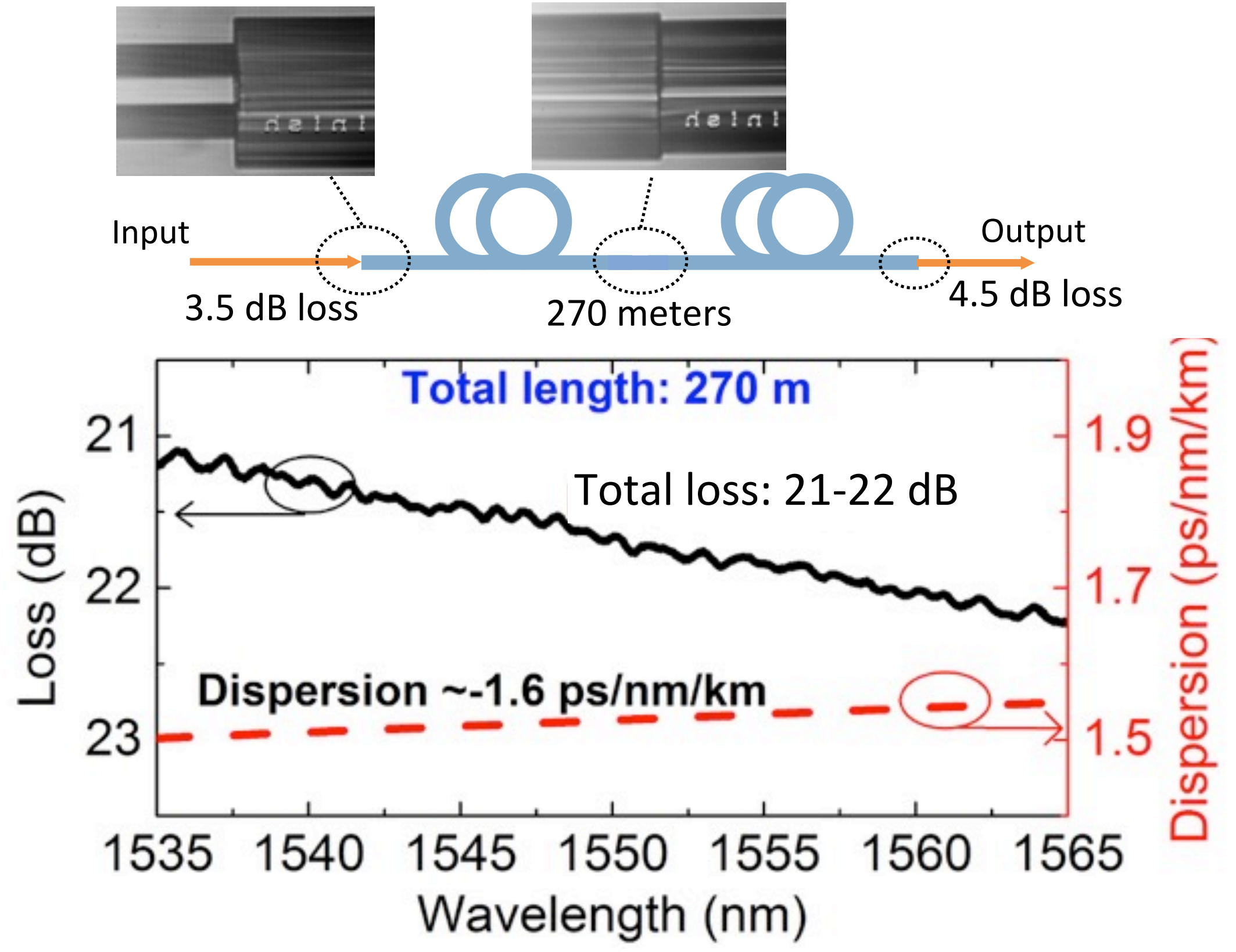




\section{Transmission Experiment: Transmitter}
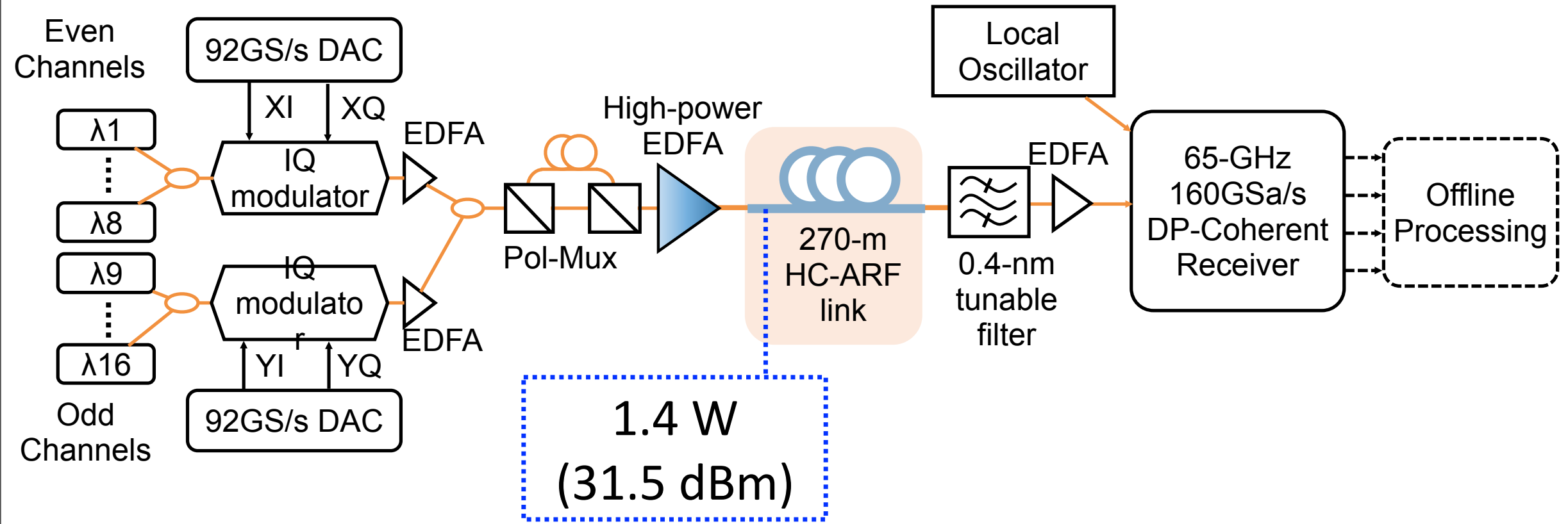

Transmitter

- $32 \mathrm{GBd}$

- 256 QAM

- EDFA output :

$3.2 \mathrm{~W}(35 \mathrm{dBm})$

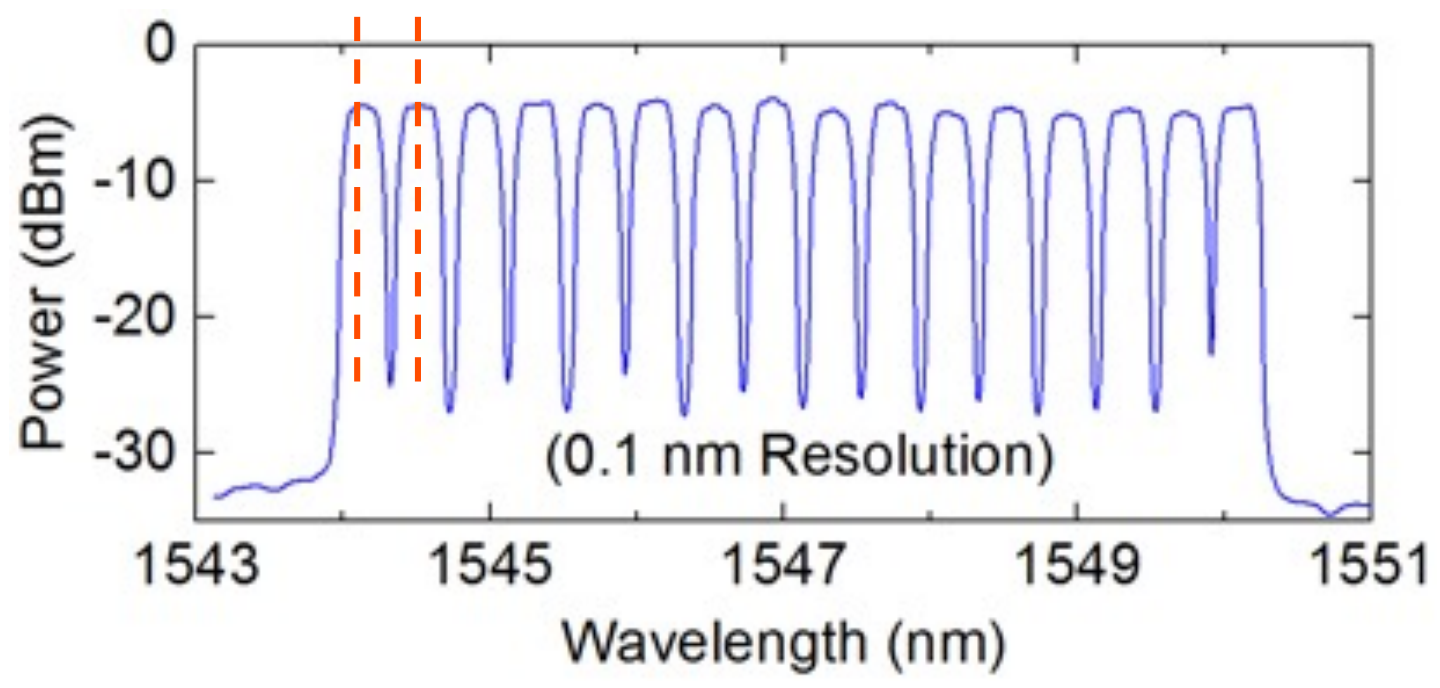




\section{WDM Transmission Results: 16-Channel 256QAM}

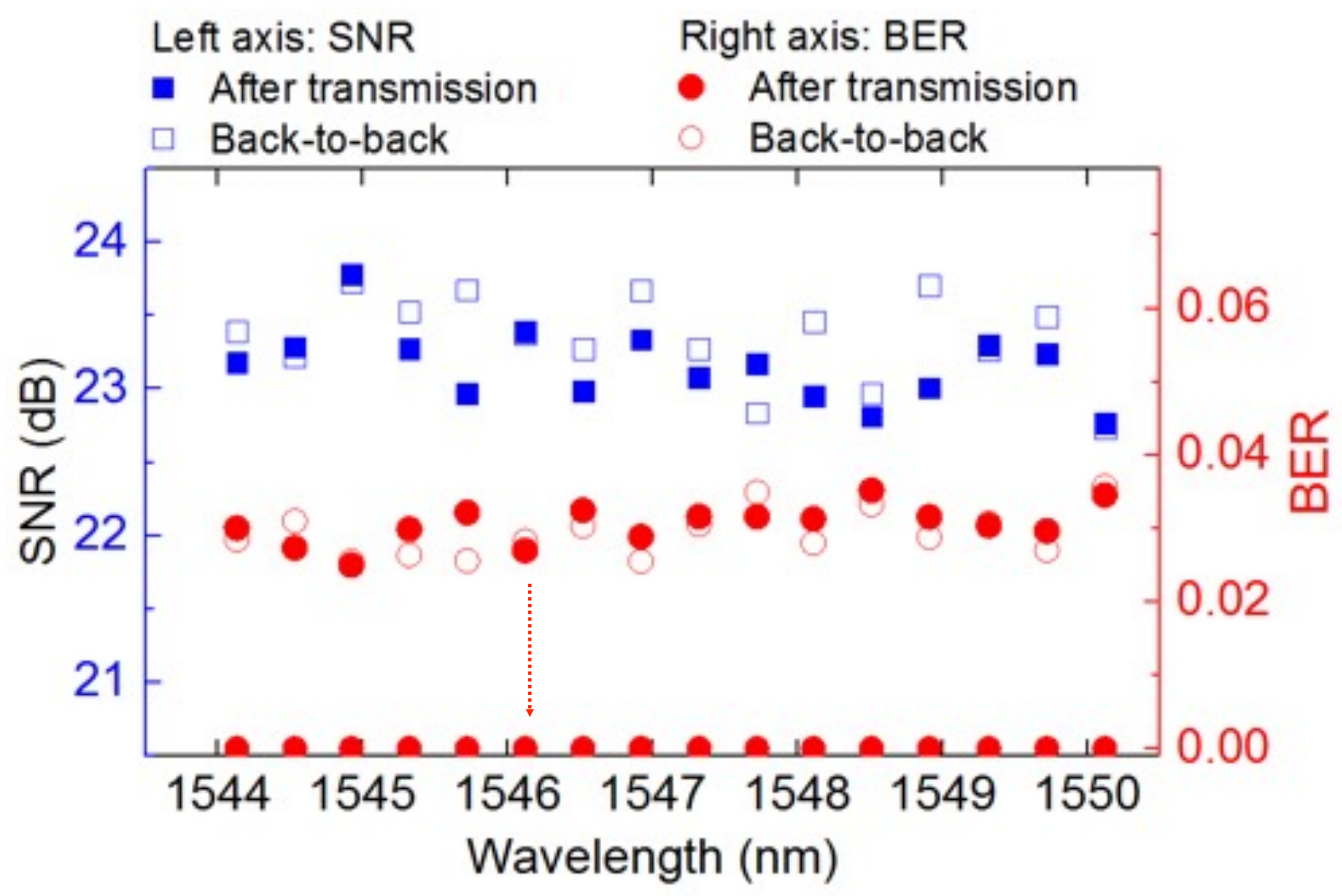

SNR: $22.8-23.7 \mathrm{~dB}$

BER: $2.7 \times 10^{-2}-3.5 \times 10^{-2}$
Error free after soft-decoding (17\% overhead IRA-LDPC) Net rate: $6.8 \mathrm{Tbit} / \mathrm{s}$ 


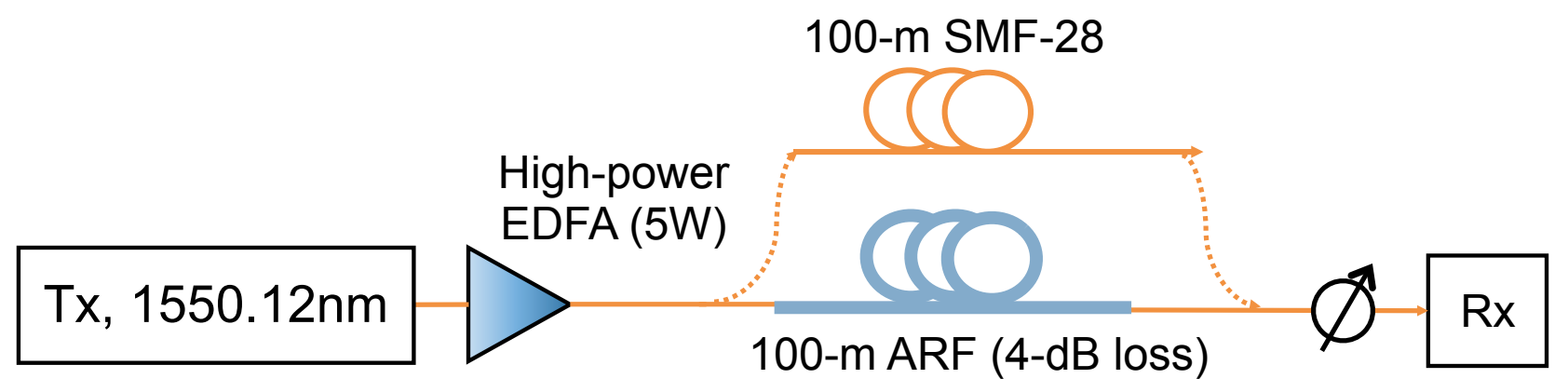

To make a comparison as fair as possible, we did:

1. Use lowest loss and short sample;

2. Compare power after transmission:

half the difference between input and output powers

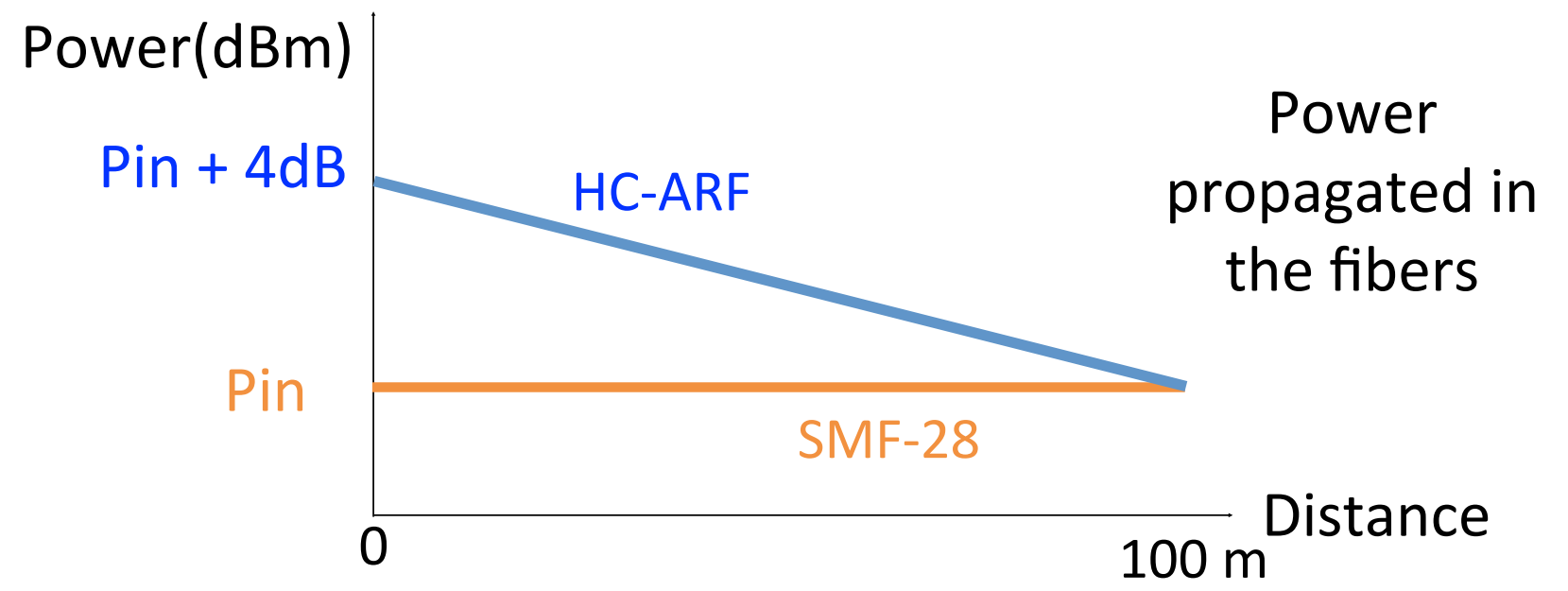

(power launched in to the hollow core fiber is

$4 \mathrm{~dB}$ higher than to SMF-28) 


\section{Single-channel Nonlinearity Tolerance: 256QAM}
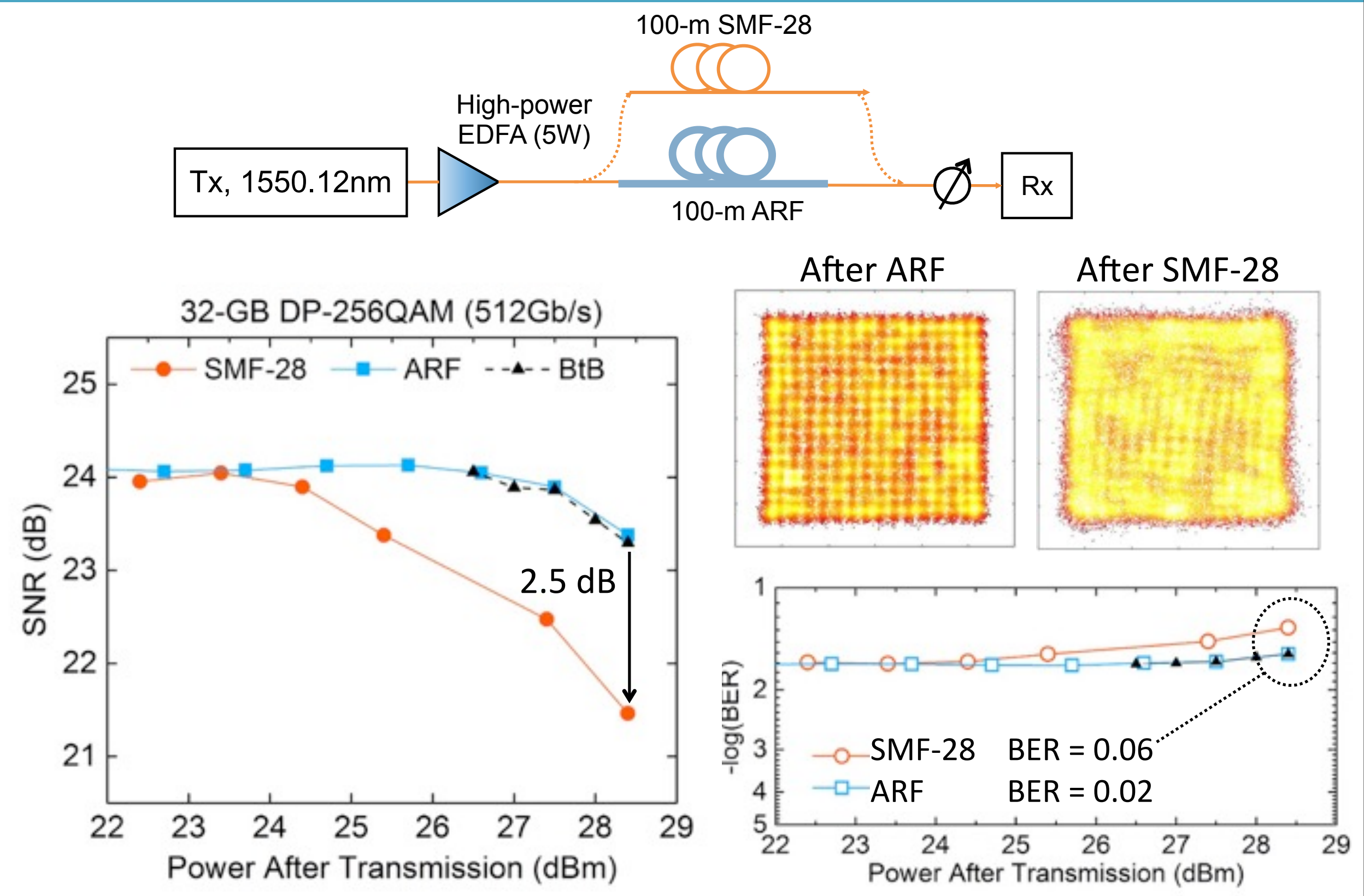


\section{Single-channel Nonlinearity Tolerance: 64QAM}

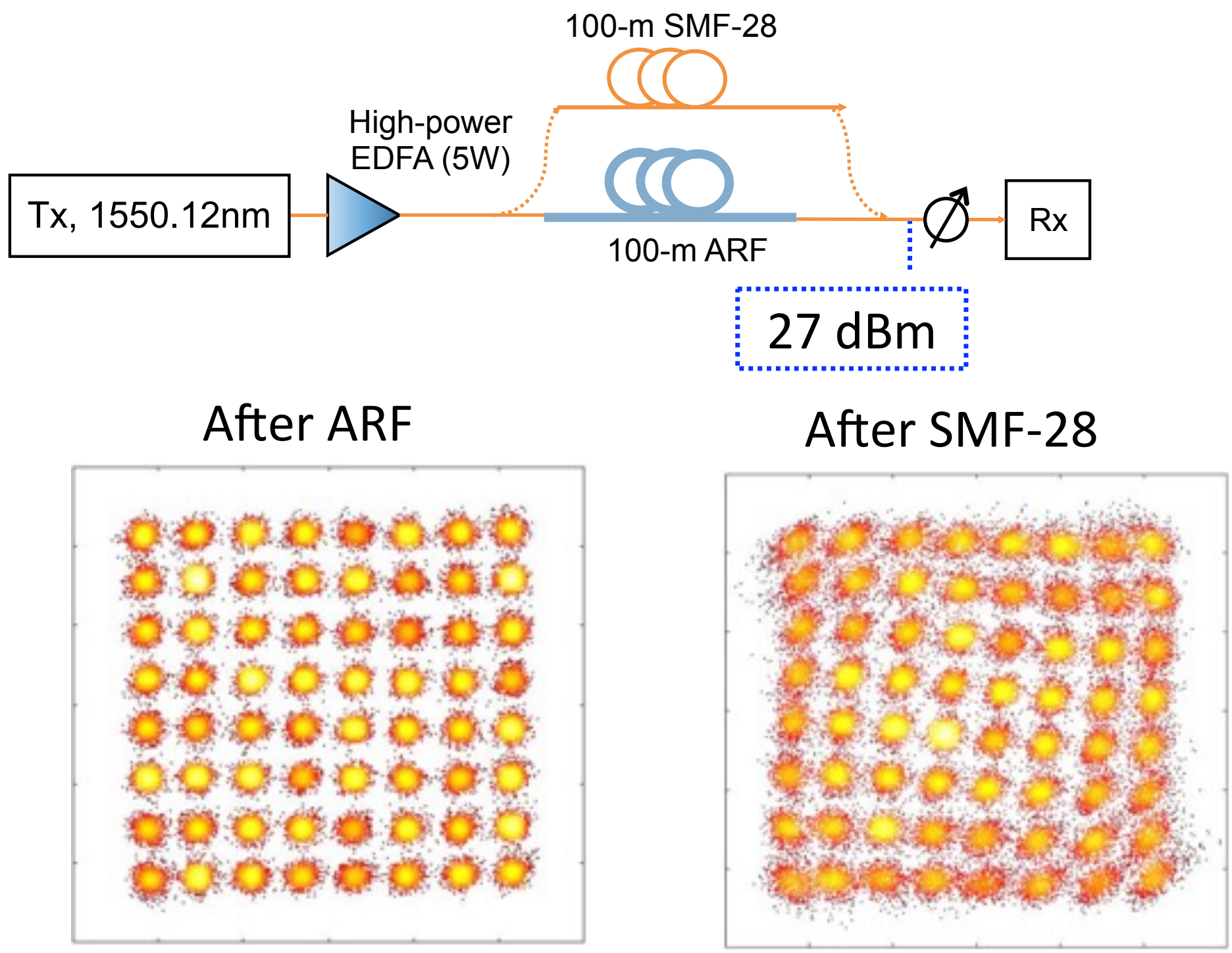




\section{INVERSTY OF \\ Southampton}

Optoelectronics

Research Centre

\section{Summary}

Using Antiresonant Fiber, we demonstrate:

- Longest transmission over HC-ARF (270 m);

- First dual-polarization coherent signal transmission;

- First WDM transmission using high-order format (2560AM);

- First comparison of signal nonlinearity penalty between SMF-28 and hollow-core fiber.

Take away message:

Low latency, low NL, HC-ARF has immediate application in inter/intra-DC links.

\section{EPSRC}

Engineering and Physical Sciences Research Council

Friday, 24 March 17
Photonic

Hyperhighway 